\title{
Matching Points with Squares
}

\author{
Bernardo M. Ábrego • Esther M. Arkin • \\ Silvia Fernández-Merchant • Ferran Hurtado • \\ Mikio Kano · Joseph S.B. Mitchell • Jorge Urrutia
}

Received: 29 July 2007 / Revised: 30 May 2008 / Accepted: 8 June 2008 / Published online: 3 July 2008

(C) Springer Science+Business Media, LLC 2008

\begin{abstract}
Given a class $\mathcal{C}$ of geometric objects and a point set $P$, a $\mathcal{C}$-matching of $P$ is a set $M=\left\{C_{1}, \ldots, C_{k}\right\} \subseteq \mathcal{C}$ of elements of $\mathcal{C}$ such that each $C_{i}$ contains exactly two elements of $P$ and each element of $P$ lies in at most one $C_{i}$. If all of the elements of $P$ belong to some $C_{i}, M$ is called a perfect matching. If, in addition, all of the elements of $M$ are pairwise disjoint, we say that this matching $M$ is strong. In this paper we study the existence and characteristics of $\mathcal{C}$-matchings for point sets in the plane when $\mathcal{C}$ is the set of isothetic squares in the plane. A consequence of our results is a proof that the Delaunay triangulations for the $L_{\infty}$ metric and the $L_{1}$ metric always admit a Hamiltonian path.
\end{abstract}

Keywords Discrete geometry $\cdot$ Matching $\cdot$ Delaunay $\cdot$ Hamiltonian

B.M. Ábrego $\cdot$ S. Fernández-Merchant

Department of Mathematics, California State University, Northridge, CA, USA

E.M. Arkin · J.S.B. Mitchell ( $\varangle)$

Department of Applied Mathematics and Statistics, State University of New York, Stony Brook, NY,

USA

e-mail: jsbm@ams.sunysb.edu

F. Hurtado

Departament de Matemàtica Aplicada II, Universitat Politècnica de Catalunya, Barcelona, Spain

M. Kano

Department of Computer and Information Sciences, Ibaraki University, Ibaraki, Japan

J. Urrutia

Instituto de Matemáticas, Universidad Nacional Autónoma de México, Mexico City, Mexico 

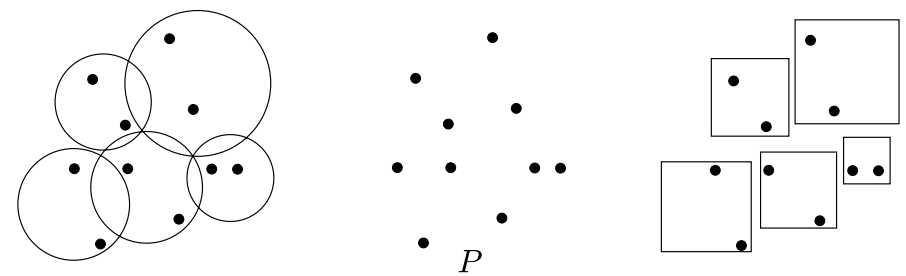

Fig. 1 A point set $P$ (center), a circle-matching of $P$ (left), and a strong square-matching of $P$ (right)

\section{Introduction}

Let $\mathcal{C}$ be a class of geometric objects, and let $P$ be a point set with an even number, $n$, of elements $p_{1}, \ldots, p_{n}$ in general position. A $\mathcal{C}$-matching of $P$ is a set $M=\left\{C_{1}, \ldots, C_{k}\right\} \subseteq \mathcal{C}$ of elements of $\mathcal{C}$ such that each $C_{i}$ contains exactly two elements of $P$ and each element of $P$ lies in at most one $C_{i}$. If all of the elements of $P$ belong to some $C_{i}, M$ is called a perfect matching. If, in addition, all of the elements of $M$ are pairwise disjoint, we say that the matching $M$ is strong.

Let $G_{\mathcal{C}}(P)$ be the graph whose vertices are the elements of $P$ and whose edges join a pair of points if there is an element of $\mathcal{C}$ containing the two points and no other points from $P$. Then, a perfect matching in $G_{\mathcal{C}}(P)$ in the usual graph theory sense corresponds to our definition of perfect $\mathcal{C}$-matching.

If $\mathcal{C}$ is the set of line segments or the set of all isothetic rectangles, then we get a segment-matching or a rectangle-matching, respectively. If $\mathcal{C}$ is the set of circles or of isothetic squares in the plane, then $M$ will be called a circle-matching or a squarematching, respectively. An example is shown in Fig. 1. Notice that these four classes of objects have in common the shrinkability property: if there is an object $C^{\prime}$ in the class that contains exactly two points $p$ and $q$ in $P$, then there is an object $C^{\prime \prime}$ in the class such that $C^{\prime \prime} \subset C^{\prime}, p$ and $q$ lie on the boundary of $C^{\prime \prime}$, and the relative interior of $C^{\prime \prime}$ is empty of points from $P$. In the case of rectangle-matchings, we can assume that the points $p$ and $q$ are at opposite corners of $C^{\prime \prime}$.

It is easy to see that $P$ always admits a strong segment-matching, i.e., a noncrossing matching in the complete geometric graph [10] induced by $P$. If no vertical or horizontal line contains two points from $P$, then $P$ necessarily admits a strong rectangle-matching, which corresponds to a noncrossing matching in the rectangleof-influence graph of $P$, in which two points are adjacent if the rectangle having them as opposite corners covers no third point from $P[8,9]$.

For the cases of circles and isothetic squares, however, the existence of matchings is not immediate, and several interesting problems arise. In this paper we study the existence of perfect and nonperfect, strong, and nonstrong square-matchings for planar point sets. In the concluding remarks we compare our results for squares with those we obtained for circle-matchings [1]. ${ }^{1}$

\footnotetext{
${ }^{1}$ A preliminary version of our results on circle-matchings and square-matchings appeared as an extended abstract in the conference paper [1].
} 
It is worth mentioning that our results on square-matchings prove, as a side effect, the fact that Delaunay triangulations for the $L_{1}$ and $L_{\infty}$ metrics contain a Hamiltonian path, a question that to the best of our knowledge remained unsolved since it was posed in the conference version of [5].

Since some of our results have quite long proofs and require several technical lemmas, for the sake of clarity of exposition, we present all of the results in Sect. 2 and present the corresponding proofs in Sect. 3.

\section{Results}

In this section, we consider geometric matchings of planar point sets using axisaligned squares. Throughout this section, we assume that no two points of $P$ lie on a common vertical or horizontal line; at the end of Sect. 3, we give detailed comments on how to handle degenerate cases.

Consider the geometric graph $G(P)$ in which the points $P$ are the vertices of $G(P)$, two of which are adjacent if there is an isothetic square containing them that does not contain another element of $P$. In other words, $G(P)$ is the Delaunay graph of $P$ in the $L_{\infty}$ metric (or the $L_{1}$ metric, if the reference is rotated 45 degrees). Under certain nondegeneracy assumptions (no four points lie on the boundary of an axisaligned square whose interior contains no point of $P), G(P)$ is a triangulation. We show that $G(P)$ always contains a perfect matching; this answers in the affirmative a question posed in the conference version of [5] (to our knowledge, this question has not previously been answered). In fact, we prove that $G(P)$ contains a Hamiltonian path; this is perhaps somewhat surprising, since it is not the case for the Euclidean $\left(L_{2}\right)$ Delaunay graph. Studying Hamiltonicity in Delaunay graphs/triangulations was the original motivation that lead Dillencourt first to find a counterexample $[4,6]$ and then subsequently to prove that Euclidean Delaunay triangulations are always 1-tough and contain perfect matchings [5]. He also proved later that deciding whether or not a Euclidean Delaunay triangulation contains a Hamiltonian cycle is NP-complete [7]. In Sect. 3 we prove:

Lemma $1 G(P)$ contains a Hamiltonian path. In particular, a Delaunay triangulation of a point set in the $L_{\infty}$ metric or the $L_{1}$ metric admits a Hamiltonian path.

By considering every other edge in a Hamiltonian path, we immediately obtain:

Theorem 1 Every planar point set $P$ of even cardinality admits a perfect squarematching.

However, a perfect strong square-matching is not always possible. An example with 10 points is shown in Fig. 3. This example can be used to construct arbitrarily large sets that do not admit perfect strong square-matchings:

Theorem 2 There are n-element point sets in the plane, for $n$ arbitrarily large, such that at most $\frac{10}{11} n$ of the $n$ points can be strongly square-matched. 
We also provide a lower bound on the fraction of points that can always be strongly square-matched:

Theorem 3 Every planar point set $P$ of $n$ points in general position has a strong square-matching using at least $2\left\lceil\frac{n}{5}\right\rceil$ points of $P$.

When the points to be matched are in convex position, one may have the intuition that a perfect strong matching always exists. This is false for circle-matchings, as we show in [1], but correct for squares, as established in the following result:

Theorem 4 Every planar point set $P$ in convex position with an even number of elements admits a perfect strong square-matching.

\section{Proofs}

\subsection{Proof of Lemma 1}

We now prove that any planar point set $P$ of even cardinality admits a perfect squarematching. In fact, we prove the stronger fact, Lemma 1, that the geometric graph $G(P)$ contains a Hamiltonian path. We start with a result, which is part of folklore, that the $L_{\infty}$ Delaunay graph in $\mathbb{R}^{2}$ is planar; we include a proof for completeness:

Lemma 2 For any planar point set $P, G(P)$ is planar.

Proof Consider two edges, $p_{i} p_{j}$ and $p_{k} p_{l}$, of $G(P)$, and let $S_{i j}$ and $S_{k l}$ be the corresponding isothetic "witness" squares, not containing other points of $P$. We claim that two edges $p_{i} p_{j}$ and $p_{k} p_{l}$ cannot cross. If $S_{i j}$ and $S_{k l}$ are disjoint, then clearly the two edges do not cross. If $S_{i j}$ and $S_{k l}$ do intersect, then their boundaries cross at two distinct points, $a$ and $b$, except in degenerate situations. The line through $a b$ separates $p_{i} p_{j}$ from $p_{k} p_{l}$, since the points $p_{i}, p_{j}$ must be on that portion of the boundary of $S_{i j}$ that does not lie inside $S_{k l}$, and similarly for $p_{k}, p_{l}$.

Now, let $C$ be a square that contains all of the elements of $P$ in its interior, and $P^{\prime}$ be the point set obtained by adding to $P$ the vertices of $C$. Let $G$ be the graph obtained from $G\left(P^{\prime}\right)$ by adding an extra point $p_{\infty}$ adjacent to the vertices of $C$. We will show that $G$ is 4-connected; before that, we prove a technical lemma.

Lemma 3 Let $Q$ be a finite point set containing the origin $O$ and a point $p$ from the first quadrant, such that all of the other points in $Q$ lie in the interior of the rectangle $R$ with corners at $O$ and $p$. Then, there is path in $G(Q)$ from $O$ to $p$ such that every two consecutive vertices can be covered by an isothetic square, empty of any other point from $Q$.

Proof The proof is by induction on $|Q|$. If $|Q|=2$, the result is obvious. If $|Q|>2$, we grow homothetically from $O$ a square with bottom left corner at $O$, until a point 
$q \in Q$, different from $O$, is found for the first time. This square gives an edge in $G(Q)$ between $O$ and $q$. Now we can apply induction to the points from $Q$ covered by the rectangle with $q$ and $p$ as opposite corners.

Clearly, the above lemma applies to any of the four quadrants with respect to any point of $P$ that is taken to be the origin.

\section{Lemma $4 G$ is 4-vertex-connected.}

Proof We argue that the graph $G^{\prime}$ resulting from the removal of any three vertices of $G$ is connected.

Suppose first that none of the removed vertices is $p_{\infty}$, and we will see that $p_{\infty}$ can be reached from any vertex $v \in G^{\prime}$. If $v$ is a corner of $C$, then it is adjacent to $p_{\infty}$. If $v$ is not such a corner, consider the four quadrants it defines. In at least one of them, no vertex from $G$ has been removed, so we can apply Lemma 3 to this quadrant and obtain a path in $G^{\prime}$ from $v$ to a surviving corner of $C$; from there we arrive at $p_{\infty}$.

If we remove from $G$ the vertex $p_{\infty}$ together with two more points in $P$, then $G^{\prime}$ contains the 4-cycle given by the corners of $C$. From any vertex $v \in P$ in $G^{\prime}$, we can reach one of these corners (and therefore any of them), since in at least two of the quadrants relative to $v$ no vertex has been removed.

The cases in which $p_{\infty}$ and one or two corners of $C$ are removed are handled similarly.

Since $G$ is planar (Lemma 2), it follows from a classic result of Tutte [11] that $G$ is Hamiltonian. This almost proves our result, since the removal of $p_{\infty}$ from $G$ results in a graph that has a Hamiltonian path. Using this path, we can now obtain a perfect matching in $G\left(P^{\prime}\right)$. A small problem remains to find a matching in $G(P)$, since the perfect matching in $G\left(P^{\prime}\right)$ may match some elements of $P$ to the corners of $C$.

To address this issue, we proceed in a way similar to that used in [3]. Consider the three shaded squares and six points $p_{1}, \ldots, p_{6}$ (represented by small circles) shown in Fig. 2. Within each of the shaded squares, place a copy of $P$, and let $P^{\prime \prime}$ be the point set containing the points of the three copies of $P$ plus $p_{1}, \ldots, p_{6}$. Consider the graph $G\left(P^{\prime \prime}\right)$ and add to it a vertex $p_{\infty}$ adjacent to $p_{1}, p_{2}, p_{3}, p_{4}$. The resulting graph is planar and 4-connected, and by Tutte's Theorem, also Hamiltonian. The removal of $p_{\infty}$ gives a Hamiltonian path $w$ in the resulting graph, with extremes in the set $\left\{p_{1}, p_{2}, p_{3}, p_{4}\right\}$. Since this path has exactly ten edges incident to points in $p_{1}, \ldots, p_{6}$, then one of the three copies of $P$ gets exactly two of these edges. Finally, all points in this copy of $P$ have to be traversed consecutively by the Hamiltonian path. This is because no point in a copy of $P$ can be adjacent to a point in another copy of $P$.

\subsection{Proof of Theorem 2}

We show first a family of 10 points that admits no perfect strong square-matching. Consider the set $P_{10}$ of 10 points, illustrated in Fig. 3: $p_{1}=(60,-2), p_{2}=(2,60)$, $q_{1}=(9,-21), q_{2}=(11,19), s_{1}=(-1,-18)$, and their symmetric points about the 
Fig. 2 The final step in the proof of existence of square-matchings

Fig. 3 Ten points that do not admit a perfect strong square-matching
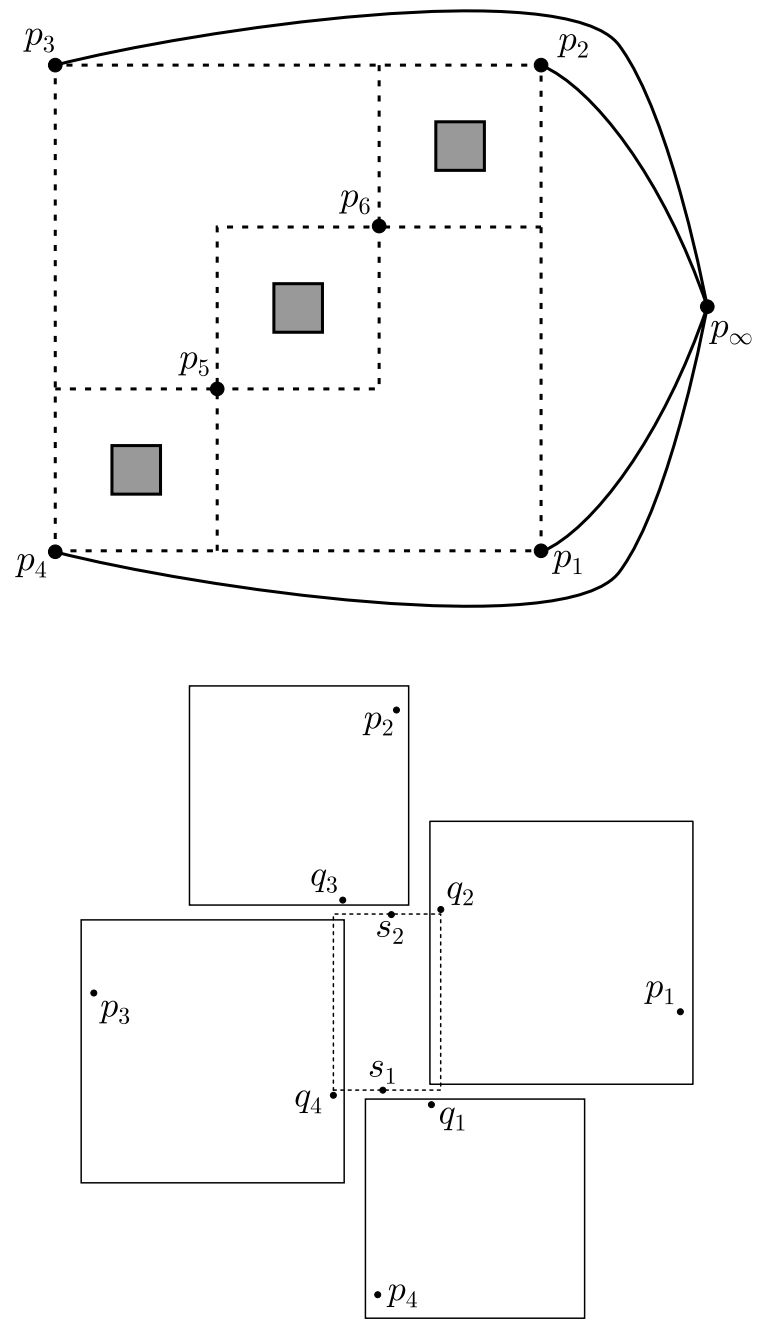

origin $p_{3}, p_{4}, q_{3}, q_{4}$, and $s_{2}$, respectively. Let $R$ denote the isothetic (dotted) rectangle with corners at the points $(11,18)$ and $(-11,-18)$.

Now, in any square matching of $P_{10}$, the point $p_{1}$ can be matched to $q_{1}$ or to $q_{2}$, but to no other point (since the corresponding bounding square would contain some other point of $P_{10}$ ). A similar observation holds for $p_{2}, p_{3}$, and $p_{4}$. Thus, in any perfect strong matching of $P_{10}$, each $p$-point must be matched to a $q$-point, forcing $s_{1}$ and $s_{2}$ to be matched. Let $S$ be the square matching $s_{1}$ to $s_{2}$. Since the vertical distance between $s_{1}$ and $s_{2}$ is 36, then $S$ has side at least 36. Since $R$ has width 22, then $S$ must contain the right side of $R$ or the left side of $R$. But the square matching $p_{1}$ (to $q_{1}$ or $q_{2}$ ) intersects the right side of $R$, and the square matching $p_{3}$ (to $q_{3}$ or $q_{4}$ ) intersects the left side of $R$, causing $S$ to intersect one of these two squares.

We now use the preceding construction to obtain arbitrarily large sets that do not admit perfect strong square-matchings, as claimed in Theorem 2. 
Fig. 4 Extending the ten-point counterexample for strong square-matchings. The small squares represent copies of the ten-point example; the other points are $(2,2),(4,4), \ldots,(2 m, 2 m)$

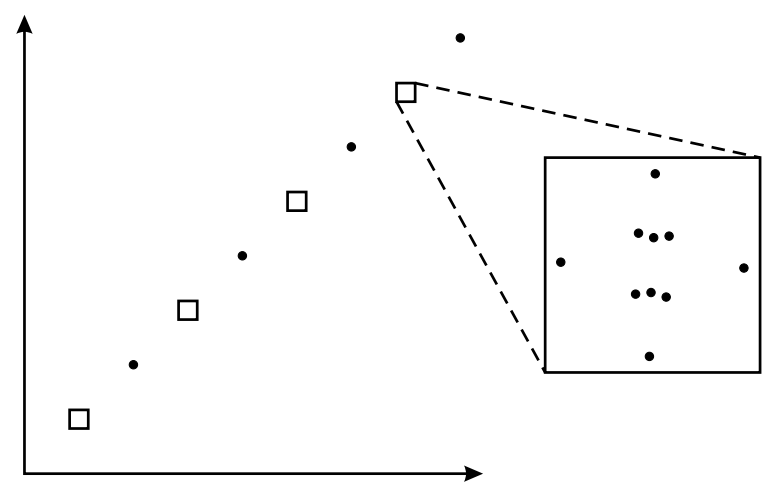

Let $n=11 m$, with $m$ even. Consider the points with coordinates $(i, i), i=$ $1, \ldots, 2 m$. For odd $i$, proceed as follows: Take a very small neighborhood of the point $(i, i)$ and replace $(i, i)$ with a copy of the ten-point configuration $P_{10}$, scaled down to fit within this $\epsilon$-neighborhood. The remaining points $(i, i)$ with even $i$ remaind singletons. Let $P$ be the point set containing all of these $10 m+m$ points, and let $M$ be a strong square-matching of $P$. See Fig. 4 .

Observe that the ten points close to the point $(1,1)$ cannot be matched among themselves. Thus, $M$ matches at most 10 of these points. This leaves two points pending. One of these points can be matched to point $(2,2)$. The remaining point cannot be matched to any point in $P$. In a similar way, one of the points in the small neighborhood of $(i, i)$ with odd $i$ cannot be matched to any element of $P$. This leaves at least $m$ elements of $P$ unmatched in $M$. Our result follows.

\subsection{Proof of Theorem 3}

We prove a result slightly stronger than Theorem 3 , from which that theorem follows immediately:

Lemma 5 Let $S$ be a square that contains a point set $P$ with $n \geq 2$ elements. Then it is always possible to find a strong square-matching of $P$ with $\left\lceil\frac{n}{5}\right\rceil$ matched pairs of points.

Proof The claim is obviously true for $n=2$. Suppose, by induction, that it is true for $n-1$, and we now prove it for $n$, with $n \geq 3$. Observe first that, if $n=5 k+i$, $i=2,3,4,5$, then $\left\lceil\frac{n}{5}\right\rceil=\left\lceil\frac{n-1}{5}\right\rceil$, and, by induction, we are done. Suppose then that $n=5 k+1$ for some $k$.

Partition $S$ into four squares $S_{1}, S_{2}, S_{3}, S_{4}$ of equal size containing $r_{1}, r_{2}, r_{3}, r_{4}$ points, respectively. If all of the $r_{i}$ 's are greater than 2 or equal to zero, we are done, since for any integers such that $r_{1}+r_{2}+r_{3}+r_{4}=n$ we have

$$
\left\lceil\frac{r_{1}}{5}\right\rceil+\cdots+\left\lceil\frac{r_{4}}{5}\right\rceil \geq\left\lceil\frac{n}{5}\right\rceil .
$$

Suppose then that some of the $r_{i}$ 's are one. A case analysis follows. 
Fig. 5 Proof of Case 1 in Lemma 5
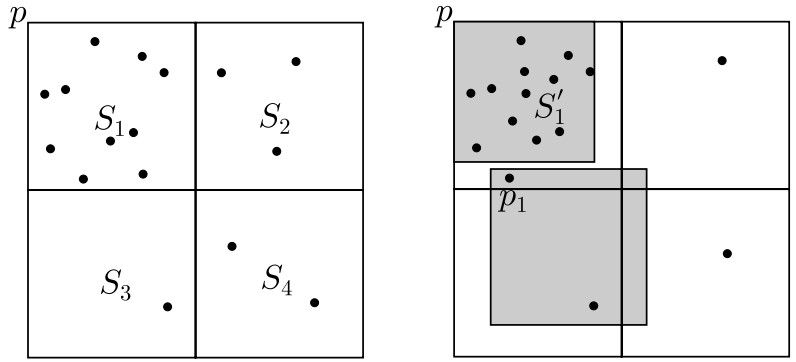

Case 1: Three elements of the set $\left\{r_{1}, r_{2}, r_{3}, r_{4}\right\}$ are equal to one (say, $r_{2}=r_{3}=$ $\left.r_{4}=1\right) ; r_{1}=5(k-1)+3$.

Let $S_{1}^{\prime}$ be the smallest square that contains all of the elements of $P$ in $S_{1}$ except one, say $p_{1}$. Let $p$ be the northwest corner of $S_{1}^{\prime}$. Suppose, without loss of generality, that $p_{1}$ lies below the horizontal line through the bottom edge of $S_{1}^{\prime}$. Then $S_{1}^{\prime}$ contains $5(k-1)+2$ points, and, thus, by induction, we can find $k$ disjoint squares in that square containing exactly two elements of $P$.

It is easy to see that there is a square contained in $S-S_{1}^{\prime}$ that contains $p_{1}$ and the element of $P$ in $S_{3}$. This square contains a square that contains exactly two elements of $P$. See Fig. 5.

Case 2: Two elements of $\left\{r_{1}, r_{2}, r_{3}, r_{4}\right\}$ are equal to one.

Suppose that $r_{i}$ and $r_{j}$ are not one. Observe that $r_{i}+r_{j}=5 k-1$ and that $\left\lceil\frac{r_{i}}{5}\right\rceil+$ $\left\lceil\frac{r_{j}}{5}\right\rceil \geq\left\lceil\frac{n-1}{5}\right\rceil=k$. If $\left\lceil\frac{r_{i}}{5}\right\rceil+\left\lceil\frac{r_{j}}{5}\right\rceil>\left\lceil\frac{n-1}{5}\right\rceil=k$, we are done. Suppose then that $\left\lceil\frac{r_{i}}{5}\right\rceil+$ $\left\lceil\frac{r_{j}}{5}\right\rceil=\left\lceil\frac{n-1}{5}\right\rceil=k$; this happens only if one of them, say $r_{i}$, is equal to $5 r$, and the other element, $r_{j}$, is equal to $5 s-1$ for some $r, s \geq 0$.

Up to symmetry, two subcases arise: (i) $r_{1}=5 r$ and $r_{3}=5 s-1$, and (ii) $r_{1}=5 r$ and $r_{4}=5 s-1$.

In case (i), let $S_{1}^{\prime}$ be the smallest square contained in $S_{1}$ that contains all but three of the elements, say $p_{1}, p_{2}$, and $p_{3}$ of $P$ in $S_{1}$, such that $p$ is a vertex of $S_{1}^{\prime}$.

If two of these elements, say $p_{1}$ and $p_{2}$, are below the horizontal line through the lower horizontal edge of $S_{1}^{\prime}$, then there is a square $S_{3}^{\prime}$ contained in $S-S_{1}^{\prime}$ that contains all of the elements of $P$ in $S_{3}$ and also contains $p_{1}$ and $p_{2}$; see Fig. 6(a). Then, by induction, we can find in $S_{1}^{\prime}$ and $S_{3}^{\prime}\left\lceil\frac{5 r-3}{5}\right\rceil=r$ and $\left\lceil\frac{5 s+1}{5}\right\rceil=s+1$ disjoint squares. Thus, we have $r+s+1=k+1$ disjoint squares contained in $S$ each of which contains exactly two elements of $P$.

If no two elements of $p_{1}, p_{2}$, and $p_{3}$ lie below the horizontal line through the lower horizontal edge of $S_{1}^{\prime}$, then there is a square contained in $S_{1} \cup S_{2}-S_{1}^{\prime}$ that contains two of these elements. See Fig. 6(b). Applying induction to the elements of $P$ in $S_{1}^{\prime}$, the elements of $P$ in $S_{3}$ and the square we just obtained proves our result. Refer to Fig. 6(b).

If $r=0$ and thus $s>0$, choose $S_{3}^{\prime}$ such that it contains all but two points of $P$ in $S_{3}$. If two points in $S_{3}$ lie above the line containing the top edge of $S_{3}^{\prime}$ or to the right of the line $L$ containing the rightmost vertical edge of $S_{3}^{\prime}$, an analysis similar 


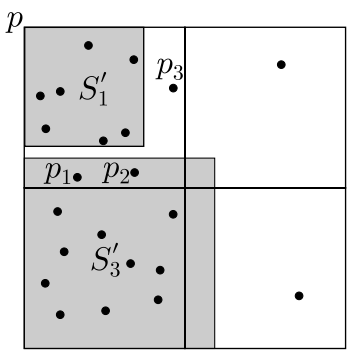

(a).

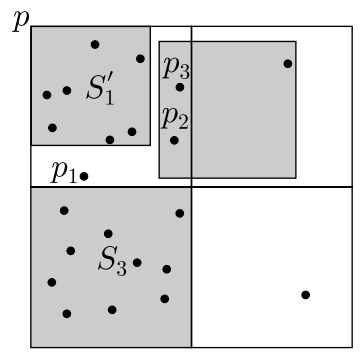

(b).

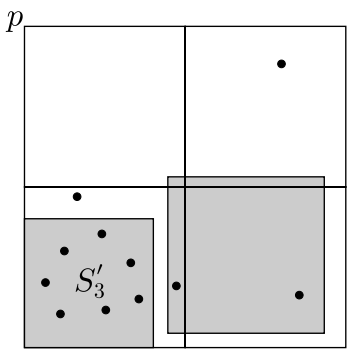

(c).

Fig. 6 Proof of Case 2 in Lemma 5

to the one above follows. Suppose then that there is exactly one point in $S_{3}$ to the right of $L$. Then $S_{3}^{\prime}$ contains $5 s-3 \geq 2$ points, and there is a square contained in $S$ containing the point of $P$ in $S_{4}$. See Fig. 6(c). By induction on the number of elements in $S_{3}^{\prime}$, and using the last square we obtained, our result follows.

Case (ii) can be handled similarly.

Case 3: Only one of $\left\{r_{1}, r_{2}, r_{3}, r_{4}\right\}$ is equal to one.

This case can be solved in a similar way to the previous cases, and we omit the details. For example, the subcase in which $r_{4}=1$ (so that $r_{1}, r_{2}$, and $r_{3}$ are multiples of 5), $r_{1} \neq 0$, and $r_{2}=0$ is solved similarly to case (i) above.

\subsection{Proof of Theorem 4}

Construction of the Matching. Consider a set $P$ of $n$ points in the plane in convex position ( $n$ even) and such that no two points lie on the same vertical or horizontal line. Label the points of $P$ from 1 to $n$ according to their counterclockwise order on the convex hull of $P$, starting with the lowest point. For ease of notation, we sometimes refer to $i, 1 \leq i \leq n$, as an integer (when it represents the label of a point in $P$ ) and sometimes as a point in the plane (an element of $P$ ); the meaning will be clear from the context. For all $i \in P$, we denote by $(i)_{x}$ and $(i)_{y}$ the $x$ - and $y$-coordinates of the point $i$. Let $S, E, N$, and $W$ be the south-, east-, north-, and west-most point in $P$, respectively (Fig. 7); we use the preceding convention for their coordinates and we even omit the brackets. It is possible that some of these points coincide. For convenience, in what follows, 1 and $n+1$ denote the same point, namely the point $S$. Assume, without loss of generality, that $S_{x}<N_{x}$. We define the regions $R_{S E}=\left\{(x, y) \in \mathbb{R}^{2}: x \geq S_{x}, y \leq E_{y}\right\}, R_{N E}=\left\{(x, y) \in \mathbb{R}^{2}: x \geq N_{x}, y \geq E_{y}\right\}$, $R_{N W}=\left\{(x, y) \in \mathbb{R}^{2}: x \leq N_{x}, y \geq W_{y}\right\}, R_{S W}=\left\{(x, y) \in \mathbb{R}^{2}: x \leq S_{x}, y \leq W_{y}\right\}$.

For $i$ and $j$ consecutive points in the convex hull of $P$, let $H(i, j)$ be the closed half-plane determined by the line joining $i$ and $j$ that contains $P$. Let $S(i, j)$ be a square containing $i$ and $j$ having the least area and the least area of intersection with $H(i, j)$ (i.e., $S(i, j)$ is a smallest-area square containing $i$ and $j$ and furthest away from $P$ ). See Fig. 7.

The length of the side of any square with least possible area containing $i$ and $j$ is equal to $l(i, j)=\max \left\{\left|(i)_{x}-(j)_{x}\right|,\left|(i)_{y}-(j)_{y}\right|\right\}$. Let

$$
C_{1}=\{S(i, i+1): 1 \leq i<n, i \text { odd }\} .
$$


Fig. 7 The cardinal regions and the squares $S(i, i+1)$

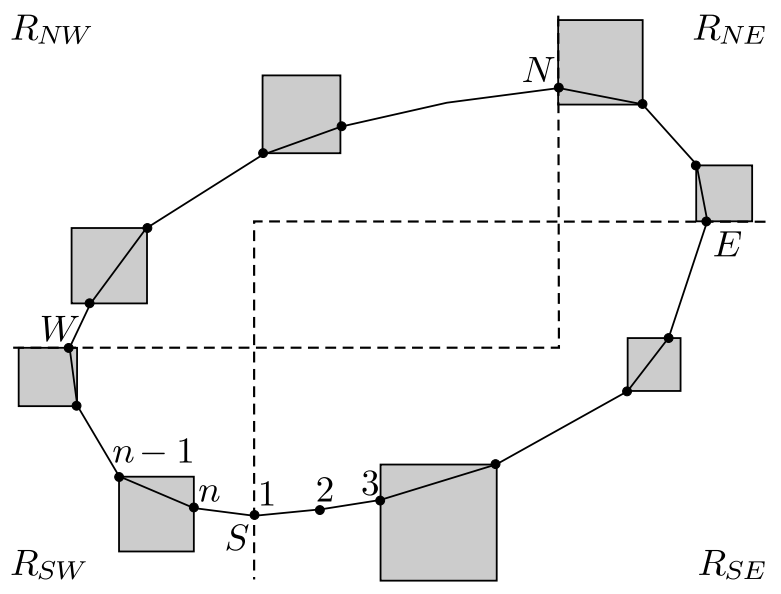

Note that, if $i, j \in R$ for some $R \in\left\{R_{S E}, R_{N E}, R_{N W}, R_{S W}\right\}$, then $S(i, j) \subseteq R$. Also, any two squares in $C_{1}$ corresponding to points in the same region do not intersect. Since $S_{x}<N_{x}$, the only two regions that may intersect are $R_{S E}$ and $R_{N W}$. Moreover, this can only happen if $W_{y}<E_{y}$. In other words, given that $S_{x}<N_{x}, C_{1}$ is a strong square-matching of $P$ if $E_{y}<W_{y}$.

Assume then that $S_{x}<N_{x}$ and $W_{y}<E_{y}$ and at least two squares in $C_{1}$ intersect. Let $S\left(p_{1}, p_{1}+1\right)$ and $S\left(q_{1}-1, q_{1}\right)$ be two squares in $C_{1}$ that intersect, and assume that such intersection is the first from left to right among elements of $C_{1}$. Formally,

$$
\begin{aligned}
& p_{1}=\min \left\{1 \leq i<n: S(i, i+1), S(j, j+1) \in C_{1} \text { intersect, for some } i<j<n\right\}, \\
& q_{1}=\max \left\{p_{1}+1<j \leq n: S(j-1, j) \in C_{1} \text { intersects } S\left(p_{1}, p_{1}+1\right)\right\} .
\end{aligned}
$$

Now we look again at consecutive squares along the boundary of the convex hull, until we find another intersection. Let

$$
C_{2}=\left\{S(i, i+1): p_{1}<i<q_{1}, i \equiv p_{1}+1(\bmod 2)\right\} .
$$

In general, for $t \geq 2$, if at least two squares in $C_{t}$ intersect, define

$$
\begin{aligned}
& p_{t}=\min \left\{p_{t-1}<i<q_{t-1}-1: S(i, i+1), S(j, j+1) \in C_{t}\right. \\
& \left.\quad \text { intersect for some } i<j<q_{t-1}-1\right\}, \\
& q_{t}=\max \left\{p_{t}+1<j<q_{t-1}: S(j-1, j) \in C_{t} \text { intersects } S\left(p_{t}, p_{t}+1\right)\right\},
\end{aligned}
$$

and

$$
C_{t+1}=\left\{S(i, i+1): p_{t}<i<q_{t}, i \equiv p_{t}+1(\bmod 2)\right\} .
$$

Let $r$ be the first $t$ such that no two squares in $C_{t}$ intersect. Note that $p_{t} \in R_{S E}$ and $q_{t} \in R_{N W}$ for all $1 \leq t \leq r$.

Now we define a second kind of square. For $i, j \in P$, and from all smallest-area squares containing $i$ and $j$, let $S^{\prime}(i, j)$ be the right-most and upper-most square. For- 


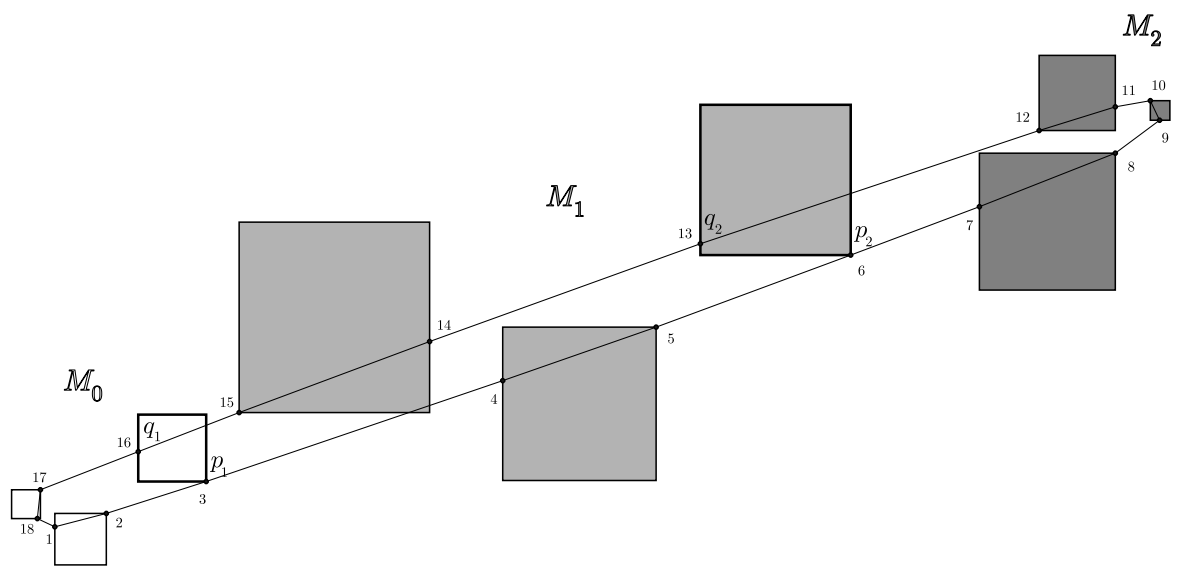

Fig. 8 The matchings $M_{t}$

mally, the lower left vertex of $S^{\prime}(i, j)$ is $\left(\min \left\{(i)_{x},(j)_{x}\right\}, \min \left\{(i)_{y},(j)_{y}\right\}\right)$, and the length of the side is $l(i, j)$.

We can now define the perfect strong matching. Consider the sets of squares

$$
M_{0}=\left\{\begin{array}{l}
\left\{S^{\prime}\left(p_{1}, q_{1}\right)\right\} \cup\left\{S(i, i+1): i \text { odd }, 1 \leq i<p_{1} \text { or } q_{1}<i<n\right\} \\
\quad \text { if } S^{\prime}\left(p_{1}, q_{1}\right) \cap S(i, i+1)=\emptyset \text { for } i \text { odd with } 1 \leq i<p_{1} \text { or } q_{1}<i<n, \\
\left\{S(i, i+1): i \text { even, } 1<i<p_{1} \text { or } q_{1} \leq i \leq n\right\} \\
\quad \text { otherwise, }
\end{array}\right.
$$

for $1 \leq t \leq r-1$,

$$
\begin{aligned}
M_{t}= & \left\{S^{\prime}\left(p_{t+1}, q_{t+1}\right)\right\} \\
& \cup\left\{S(i, i+1): i \equiv p_{t}+1(\bmod 2), p_{t}<i<p_{t+1} \text { or } q_{t+1}<i<q_{t}\right\},
\end{aligned}
$$

and

$$
M_{r}=C_{r}=\left\{S(i, i+1): i \equiv p_{r}+1(\bmod 2) \text { and } p_{r}<i<q_{r}\right\} .
$$

Define $M=\bigcup_{t=0}^{r} M_{t}$. Observe that every point in $P$ belongs to some square in $M$. The following lemmas will be used later to prove that $M$ is a strong perfect matching.

Technical Lemmas. Note that any line joining two points both in $R_{S E}$ or both in $R_{N W}$ has positive slope. Then

$$
(i)_{x}<(j)_{x} \text { and }(i)_{y}<(j)_{y} \quad \text { if } i<j \text { and } i, j \in R_{S E} \text {, }
$$

and

$$
(i)_{x}>(j)_{x} \text { and }(i)_{y}>(j)_{y} \quad \text { if } i<j \text { and } i, j \in R_{N W} \text {. }
$$


In particular, for all $1 \leq t \leq r$, since $p_{t}, p_{t}+1 \in R_{S E}$ and $q_{t}, q_{t}+1 \in R_{N W}$, we have

$$
\left(p_{t}\right)_{x}<\left(p_{t}+1\right)_{x} \quad \text { and } \quad\left(p_{t}\right)_{y}<\left(p_{t}+1\right)_{y}
$$

and

$$
\left(q_{t}\right)_{x}<\left(q_{t}-1\right)_{x} \text { and }\left(q_{t}\right)_{y}<\left(q_{t}-1\right)_{y} .
$$

It turns out that we can guarantee other similar order relationships among $p_{t}-1, p_{t}$, $p_{t}+1$ and $q_{t}-1, q_{t}, q_{t}+1$.

Lemma 6 For any $1 \leq t \leq r$, we have that

$$
\begin{aligned}
& \left(p_{t}\right)_{x}<\left(q_{t}-1\right)_{x} \quad \text { and } \quad\left(p_{t}\right)_{y}<\left(q_{t}-1\right)_{y}, \\
& \left(q_{t}\right)_{x}<\left(p_{t}+1\right)_{x} \quad \text { and } \quad\left(q_{t}\right)_{y}<\left(p_{t}+1\right)_{y},
\end{aligned}
$$

and if $t \geq 2$,

$$
\left(q_{t}+1\right)_{x}<\left(p_{t}\right)_{x} \text { and }\left(p_{t}-1\right)_{y}<\left(q_{t}\right)_{y} .
$$

Proof Since $q_{t}-1, q_{t} \in R_{N W}$, the square $S\left(q_{t}-1, q_{t}\right)$ is completely to the left of the vertical line $x=\max \left\{\left(q_{t}-1\right)_{x},\left(q_{t}\right)_{x}\right\}=\left(q_{t}-1\right)_{x}$ and $S\left(p_{t}, p_{t}+1\right)$ is completely to the right of the vertical line $x=\min \left\{\left(p_{t}\right)_{x},\left(p_{t}+1\right)_{x}\right\}=\left(p_{t}\right)_{x}$. Since $S\left(p_{t}, p_{t}+1\right)$ $\cap S\left(q_{t}-1, q_{t}\right) \neq \emptyset$, we must have that

$$
\left(p_{t}\right)_{x}<\left(q_{t}-1\right)_{x} .
$$

We also know that $q_{t}-1$ belongs to

$$
H\left(p_{t}, p_{t}+1\right)=\left\{(x, y): y \geq m\left(x-\left(p_{t}\right)_{x}\right)+\left(p_{t}\right)_{y}\right\},
$$

where $m=\left(\left(p_{t}+1\right)_{y}-\left(p_{t}\right)_{y}\right) /\left(\left(p_{t}+1\right)_{x}-\left(p_{t}\right)_{x}\right)>0$. Thus,

$$
\left(q_{t}-1\right)_{y} \geq m\left(\left(q_{t}-1\right)_{x}-\left(p_{t}\right)_{x}\right)+\left(p_{t}\right)_{y}>m\left(\left(p_{t}\right)_{x}-\left(p_{t}\right)_{x}\right)+\left(p_{t}\right)_{y}=\left(p_{t}\right)_{y} .
$$

This proves (5). The proof of (6) is similar. To prove the second inequality in (7), assume by contradiction that $\left(q_{t}\right)_{y} \leq\left(p_{t}-1\right)_{y}$ for some $t \geq 2$. Then $p_{t-1}$ is defined and $S \leq p_{t-1} \leq p_{t}-1$, so both $p_{t}-1$ and $p_{t}$ are in $R_{S E}$. Our assumption, together with (1) and (5), gives

$$
\begin{aligned}
& \left(q_{t}\right)_{y} \leq\left(p_{t}-1\right)_{y}<\left(p_{t}\right)_{y}<\left(q_{t}-1\right)_{y} \quad \text { and } \\
& \left(p_{t}-1\right)_{x}<\left(p_{t}\right)_{x}<\left(q_{t}-1\right)_{x} .
\end{aligned}
$$

Also, $p_{t}-1$ belongs to $H\left(q_{t}-1, q_{t}\right)$ (see Fig. 9). Hence, $p_{t}-1$ belongs to the right triangle in $H\left(q_{t}-1, q_{t}\right)$ bounded by the segment $q_{t}\left(q_{t}-1\right)$ and the lines $x=$ $\left(q_{t}-1\right)_{x}$ and $y=\left(q_{t}\right)_{y}$. That is, the point $p_{t}-1$ is in the interior of $S\left(q_{t}-1, q_{t}\right)$. If $p_{t-1}<p_{t}-1$, then since $p_{t-1}$ and $p_{t}$ have different parity, $p_{t-1}<p_{t}-2<p_{t}<$ $q_{t}-1$. Moreover, $S\left(p_{t}-2, p_{t}-1\right) \in C_{t}$ intersects $S\left(q_{t}-1, q_{t}\right)$, which contradicts 
Fig. 9 Proof of Lemma 6

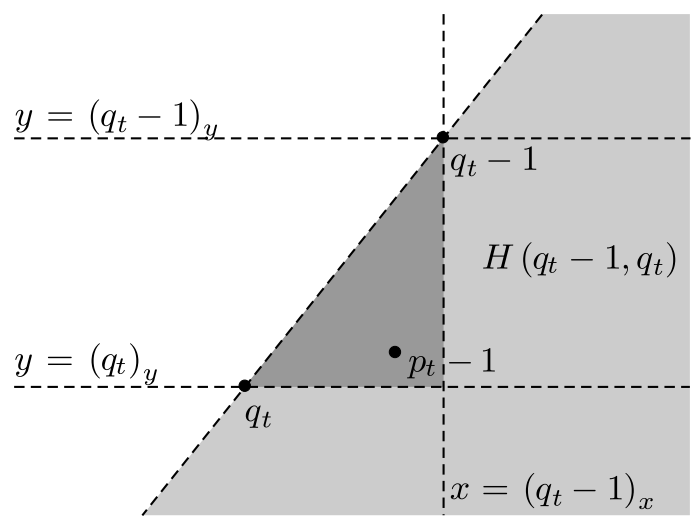

the definition of $p_{t}$. If, on the other hand, $p_{t-1}=p_{t}-1$, then by (4), (2), our previous observation, and (3) we have

$$
\left(q_{t-1}\right)_{x} \leq\left(q_{t-1}-1\right)_{x} \leq\left(q_{t}\right)_{x}<\left(p_{t}-1\right)_{x}=\left(p_{t-1}\right)_{x}<\left(p_{t-1}+1\right)_{x},
$$

which means that $S\left(q_{t-1}-1, q_{t-1}\right)$ and $S\left(p_{t-1}, p_{t-1}+1\right)$ are separated by the vertical line $x=\left(q_{t}\right)_{x}$, i.e., $S\left(q_{t-1}-1, q_{t-1}\right) \cap S\left(p_{t-1}, p_{t-1}+1\right)=\emptyset$, a contradiction. The proof of the first inequality in (7) is similar.

Lemma 7 For $1 \leq t \leq r$, define

$$
R^{+}(t)=\left\{(x, y): x>\max \left\{\left(p_{t}\right)_{x},\left(q_{t}\right)_{x}\right\} \text { and } y>\max \left\{\left(p_{t}\right)_{y},\left(q_{t}\right)_{y}\right\}\right\} .
$$

Then, for all $i$ such that $p_{t}+1 \leq i \leq q_{t}-1$, we have that $i \in R^{+}(t)$.

Proof We know that $S \leq p_{t}<p_{t}+1 \leq E$ and $N \leq q_{t}-1<q_{t} \leq W$. So for all $i$ such that $p_{t}+1 \leq i \leq q_{t}-1$, we have that

$$
i \in\left\{(x, y): x \geq \min \left\{\left(p_{t}+1\right)_{x},\left(q_{t}-1\right)_{x}\right\} \text { and } y \geq \min \left\{\left(p_{t}+1\right)_{y},\left(q_{t}-1\right)_{y}\right\}\right\} .
$$

Indeed, if $p_{t}+1 \leq i \leq E$, then $(i)_{x} \geq\left(p_{t}+1\right)_{x}$ and $(i)_{y} \geq\left(p_{t}+1\right)_{y}$ by (1), if $E \leq i \leq N$, then $(i)_{x} \geq N_{x} \geq\left(q_{t}-1\right)_{x}$ and $(i)_{y} \geq E_{y} \geq\left(p_{t}+1\right)_{y}$, and if $N \leq i \leq q_{t}-1$, then $(i)_{x} \geq\left(q_{t}-1\right)_{x}$ and $(i)_{y} \geq\left(q_{t}-1\right)_{y}$ by (2). Therefore it is enough to show that both $p_{t}+1$ and $q_{t}-1$ belong to $R^{+}(t)$. But (3) and (6) imply that $\left(p_{t}+1\right)_{x}>\max \left\{\left(p_{t}\right)_{x},\left(q_{t}\right)_{x}\right\}$ and $\left(p_{t}+1\right)_{y}>\max \left\{\left(p_{t}\right)_{y},\left(q_{t}\right)_{y}\right\}$; and (4) and (5) imply that $\left(q_{t}-1\right)_{x}>\max \left\{\left(p_{t}\right)_{x},\left(q_{t}\right)_{x}\right\}$ and $\left(q_{t}-1\right)_{y}>\max \left\{\left(p_{t}\right)_{y},\left(q_{t}\right)_{y}\right\}$. So $\left\{p_{t}+1, q_{t}-1\right\} \subseteq R^{+}(t)$.

Lemma 8 If $p_{t}+1 \leq i<E$, then $S(i, i+1) \subseteq R^{+}(t)$ or $S(i, i+1)$ is completely to the right of $S^{\prime}\left(p_{t}, q_{t}\right)$, that is,

$$
S(i, i+1) \subseteq\left\{(x, y): x \geq \min \left\{\left(p_{t}\right)_{x},\left(q_{t}\right)_{x}\right\}+l\left(p_{t}, q_{t}\right)\right\} .
$$

If $N \leq i<q_{t}-1$, then $S(i, i+1) \subseteq R^{+}(t)$ or $S(i, i+1)$ is completely above 


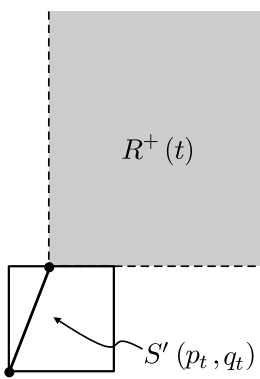

(a)

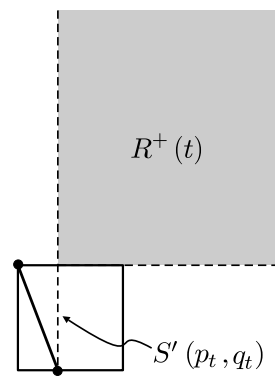

(b)

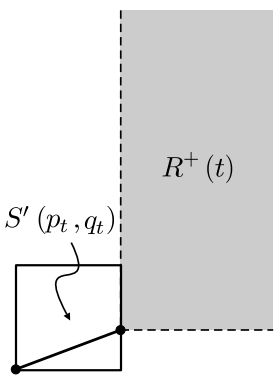

(c)

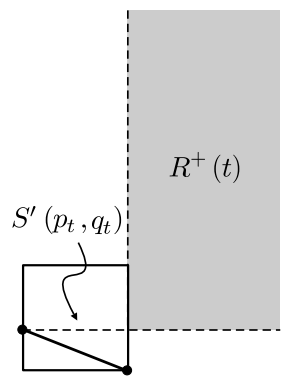

(d)

Fig. 10 The region $R^{+}(t)$

$S^{\prime}\left(p_{t}, q_{t}\right)$, that is,

$$
S(i, i+1) \subseteq\left\{(x, y): y \geq \min \left\{\left(p_{t}\right)_{y},\left(q_{t}\right)_{y}\right\}+l\left(p_{t}, q_{t}\right)\right\} .
$$

Proof We denote by slope $(i, j)$ the slope of the line passing through the points $i$ and $j$. Assume first that $p_{t}+1 \leq i<E$. Then, by Lemma 7 we have that $i$ and $i+1$ are in $R^{+}(t)$. By the definition of $S(i, i+1)$, when $i$ and $i+1$ are in $R_{S E}$, we have that $S(i, i+1) \subseteq\left\{(x, y): x \geq(i)_{x}\right\}$. Hence, if $\left|\operatorname{slope}\left(p_{t}, q_{t}\right)\right| \leq 1$ (see Figs. 10(c) and (d)), then (8) holds. Also, if slope $(i, i+1) \geq 1$, then $S(i, i+1) \subseteq R^{+}(t)$.

Assume then that slope $(i, i+1)<1$ and $\left|\operatorname{slope}\left(p_{t}, q_{t}\right)\right|>1$ (Figs. 10(a) and (b)). Since $S \leq p_{t}<p_{t}+1 \leq i<i+1 \leq E$, then by convexity $\operatorname{slope}\left(p_{t}, p_{t}+1\right)<$ slope $(i, i+1)<1$. Consider the points $u$ and $v$ given by the intersection of the lines with slope -1 or 1 passing through $p_{t}$ and the horizontal line passing through $p_{t}+1$ (Fig. 11). Since $q_{t} \in H\left(p_{t}, p_{t}+1\right)$, $\left|\operatorname{slope}\left(p_{t}, q_{t}\right)\right|>1$, and (6) holds, we have that $q_{t}$ belongs to the interior of the triangle $u p_{t} v$. Hence, $\min \left\{\left(p_{t}\right)_{x},\left(q_{t}\right)_{x}\right\}+l\left(p_{t}, q_{t}\right) \leq$ $(v)_{x} \leq\left(p_{t}+1\right)_{x} \leq(i)_{x}$, and (8) holds. The proof of (9) is similar.

Lemma 9 For $1 \leq t \leq r$, we have the following:

1. If $1 \leq i \leq p_{t}-1$, then $i \in R_{\text {down }}^{-}(t)=\left\{(x, y): y<\min \left\{\left(p_{t}\right)_{y},\left(q_{t}\right)_{y}\right\}\right\}$.

2. If $q_{t}+1 \leq i \leq W$, then $i \in R_{\text {left }}^{-}(t)=\left\{(x, y): x<\min \left\{\left(p_{t}\right)_{x},\left(q_{t}\right)_{x}\right\}\right\}$.

3. If $t \geq 2$ and $W \leq i \leq n+1$, then $i \in R_{\mathrm{down}}^{-}(t) \cap R_{\text {left }}^{-}(t)$.

(Here, $n+1$ and 1 represent the same point.)

Proof Consider $1 \leq i \leq p_{t}-1$. If such $i$ exists, then $S \leq p_{t}-1<p_{t}<E$ and so $\left(p_{t}-1\right)_{y}<\left(p_{t}\right)_{y}$. This and (7) imply that $\left(p_{t}-1\right)_{y}<\min \left\{\left(p_{t}\right)_{y},\left(q_{t}\right)_{y}\right\}$. So, if $1 \leq i \leq p_{t}-1$, then $(i)_{y} \leq\left(p_{t}-1\right)_{y}<\min \left\{\left(p_{t}\right)_{y},\left(q_{t}\right)_{y}\right\}$. Similarly, if $N \leq q_{t}<$ $q_{t}+1 \leq q_{t-1} \leq W$, then $\left(q_{t}+1\right)_{x}<\left(q_{t}\right)_{x}$. Also by (7) $\left(q_{t}+1\right)_{x}<\left(p_{t}\right)_{x}$. So, if $q_{t}+1 \leq i \leq W$, then $(i)_{x} \leq\left(q_{t}+1\right)_{x}<\min \left\{\left(p_{t}\right)_{x},\left(q_{t}\right)_{x}\right\}$. Finally, if $t \geq 2$, then $p_{t-1}$ and $q_{t-1}$ are defined. Note that since $N \leq q_{t}<q_{t-1} \leq W, W_{y} \leq\left(q_{t}\right)_{y}$ by (2), and $W_{y} \leq\left(q_{t-1}\right)_{y}<\left(p_{t-1}+1\right)_{y}<\left(p_{t}\right)_{y}$ by (6). So, if $W \leq i \leq n$, then $(i)_{y} \leq W_{y}<$ $\min \left\{\left(p_{t}\right)_{y},\left(q_{t}\right)_{y}\right\}$. Also, $S_{x}<\left(p_{t}\right)_{x}$ and by (5), $S_{x} \leq\left(p_{t-1}\right)_{x}<\left(q_{t-1}-1\right)_{x}<\left(q_{t}\right)_{x}$. So, if $W \leq i \leq n$, then $(i)_{x} \leq S_{x}<\min \left\{\left(p_{t}\right)_{x},\left(q_{t}\right)_{x}\right\}$. 


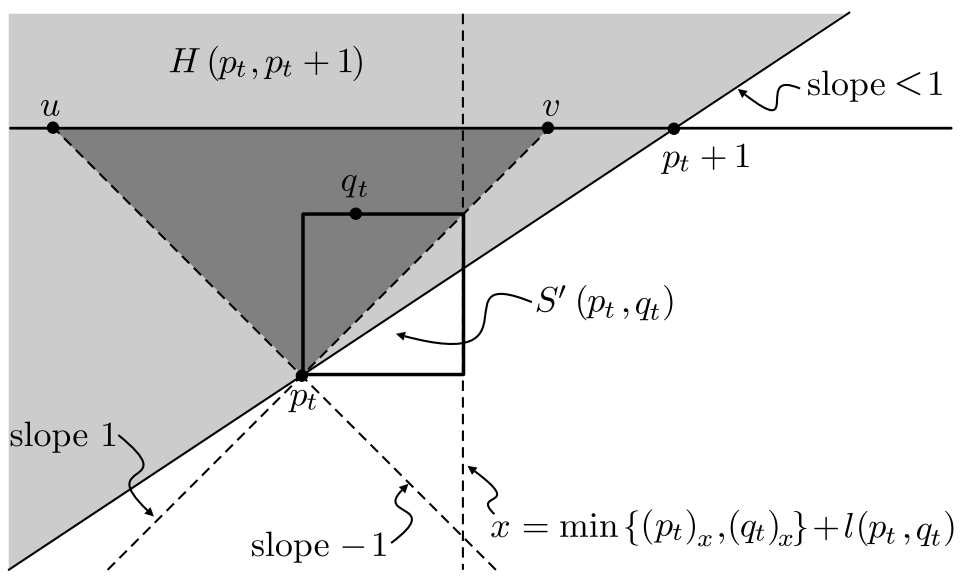

Fig. 11 Proof of Lemma 8

Proof that $M$ is a Strong Square-Matching of $P$. The proof follows from the following three claims:

Claim 1 If $1 \leq t<u \leq r$, then $S^{\prime}\left(p_{t}, q_{t}\right) \cap S^{\prime}\left(p_{u}, q_{u}\right)=\emptyset$.

Proof Assume that $1 \leq t<u \leq r$. Then $p_{t}+1 \leq p_{u}<q_{u} \leq q_{t}-1$, and, by Lemma 7, $p_{u} q_{u} \in R^{+}(t)$. Thus, by the definition of $S^{\prime}\left(p_{u}, q_{u}\right)$ we have $S^{\prime}\left(p_{u}, q_{u}\right) \subseteq R^{+}(t)$. On the other hand, by the definition of $R^{+}(t)$ we have $S^{\prime}\left(p_{t}, q_{t}\right) \cap R^{+}(t)=\emptyset$. Therefore $S^{\prime}\left(p_{t}, q_{t}\right) \cap S^{\prime}\left(p_{u}, q_{u}\right)=\emptyset($ see Fig. 10).

Claim 2 If $S(i, i+1), S^{\prime}\left(p_{t}, q_{t}\right) \in M$, then $S(i, i+1) \cap S^{\prime}\left(p_{t}, q_{t}\right)=\emptyset$.

Proof Assume that $S(i, i+1) \in M$. If $p_{t}+1 \leq i<i+1 \leq E$ or $N \leq i<i+1 \leq$ $q_{t}-1$, then the result follows from Lemma 8. If $E \leq i<i+1 \leq N$, then $S(i, i+1) \subseteq$ $R_{N E}$. Also, by Lemma 7, both $N$ and $E$ are in $R^{+}(t)$. Thus, $R_{N E} \subseteq R^{+}(t)$. Since $R^{+}(t) \cap S^{\prime}\left(p_{t}, q_{t}\right)=\emptyset$, the result holds.

If $S^{\prime}\left(p_{1}, q_{1}\right) \in M$, then, by the definition of $M_{0}, S^{\prime}\left(p_{1}, q_{1}\right)$ does not intersect $S(i, i+1)$ for all $1 \leq i<p_{1}$ or $q_{1}<i<n$. Assume then that $t \geq 2$. Hence, by Lemma 9, if $1 \leq i<p_{t}-1$, then $i, i+1 \in R_{\text {down }}^{-}(t)$, and, since $i, i+1 \in R_{S E}$, $S(i, i+1) \subseteq R_{\text {down }}^{-}(t)$. If $q_{t}-1 \leq i<W$, then $i, i+1 \in R_{\text {left }}^{-}(t)$, and, since $i, i+$ $1 \in R_{N W}, S(i, i+1) \subseteq R_{\text {left }}^{-}(t)$. Also, by Lemma 9 , since $t \geq 2, S$ and $W$ are in $R_{\text {down }}^{-}(t) \cap R_{\text {left }}^{-}(t)$. Hence, if $W \leq i \leq n+1$, then $S(i, i+1) \subseteq R_{S W} \subseteq R_{\text {down }}^{-}(t) \cap$ $R_{\text {left }}^{-}(t)$. Finally, note that $S^{\prime}\left(p_{t}, q_{t}\right) \cap\left(R_{\text {down }}^{-}(t) \cup R_{\text {left }}^{-}(t)\right)=\emptyset$, and, thus, in all cases the result holds.

Claim 3 If $S(i, i+1) \neq S(j, j+1)$ are in $M$, then $S(i, i+1) \cap S(j, j+1)=\emptyset$.

Proof The result is true if $i, i+1, j, j+1$ all belong to the same region $R \in$ $\left\{R_{S E}, R_{N E}, R_{N W}, R_{S W}\right\}$, or if $i, i+1 \in R_{S W}$ or $j, j+1 \in R_{N E}$. Assume that 
$S(i, i+1) \in M_{t}$ and $S(j, j+1) \in M_{u}$ for some $0 \leq t \leq u \leq r$ and one of the pairs $\{i, i+1\}$ or $\{j, j+1\}$ is contained in $R_{S E}$ and the other in $R_{N W}$.

If $t<u$, we show that $S^{\prime}\left(p_{u}, q_{u}\right)$ "separates" $S(i, i+1)$ and $S(j, j+1)$. In this case, we have that either

$$
p_{u}<j<j+1 \leq E \quad \text { and } \quad q_{u}<i<i+1 \leq W
$$

or

$$
N \leq j<j+1<q_{u} \text { and } 1 \leq i<i+1<p_{u} .
$$

Then, by Lemma 7, $j$ and $j+1$ are in $R^{+}(u)$. Moreover, if (10) holds, then Lemma 8 implies that

$$
S(j, j+1) \subseteq R^{+}(u) \cup\left\{(x, y): x>\min \left\{\left(p_{u}\right)_{x},\left(q_{u}\right)_{x}\right\}\right\}
$$

Also, by Lemma 9, $S(i, i+1) \subseteq R_{\text {left }}^{-}(u)$. Since $R^{+}(u) \cup\left\{(x, y): x>\min \left\{\left(p_{u}\right)_{x}\right.\right.$, $\left.\left.\left(q_{u}\right)_{x}\right\}\right\}$ and $R_{\text {left }}^{-}(u)$ are disjoint, $S(i, i+1) \cap S(j, j+1)=\emptyset$. Similarly, if (11) holds, then, by Lemma 8 ,

$$
S(j, j+1) \subseteq R^{+}(u) \cup\left\{(x, y): y>\min \left\{\left(p_{u}\right)_{y},\left(q_{u}\right)_{y}\right\}\right\} .
$$

Additionally, by Lemma $9, S(i, i+1) \subseteq R_{\text {down }}^{-}(u)$. Since $R^{+}(u) \cup\{(x, y): y>$ $\left.\min \left\{\left(p_{u}\right)_{y},\left(q_{u}\right)_{y}\right\}\right\}$ and $R_{\text {down }}^{-}(u)$ are disjoint, $S(i, i+1) \cap S(j, j+1)=\emptyset$.

Now if $t=u \neq 0$, then the result is true by the definition of $p_{t+1}$ and $q_{t+1}$ if $t<r$ or by the definition of $r$ if $t=r$. Finally, if $t=u=0$, then we have two cases. First, if $S^{\prime}\left(p_{1}, q_{1}\right) \in M$, then $S(i, i+1), S(j, j+1) \in C_{1}$ and therefore, by the definition of $p_{1}$ and $q_{1}, S(i, i+1) \cap S(j, j+1)=\emptyset$. Second, if $S^{\prime}\left(p_{1}, q_{1}\right) \notin M$, then $i$ and $j$ are even and there is an odd $k$ such that $1 \leq k<p_{1}$ or $q_{1}+1 \leq k<n$ and $S(k, k+1) \cap S^{\prime}\left(p_{1}, q_{1}\right) \neq \emptyset$. By Lemma 9 (parts 1 and 2), if $1 \leq k<p_{1}$ or $q_{1}+1 \leq k<W$, then $S(k, k+1) \subseteq R_{\text {down }}^{-}(1) \cup R_{\text {left }}^{-}(1)$ but $R_{\text {down }}^{-}(1) \cup R_{\text {left }}^{-}(1)$ and $S^{\prime}\left(p_{1}, q_{1}\right)$ are disjoint, so $S(k, k+1)$ and $S^{\prime}\left(p_{1}, q_{1}\right)$ would also be disjoint. Hence, $W \leq k<n$ and so $S(k, k+1) \subseteq R_{S W}$. Since $S(k, k+1) \cap S^{\prime}\left(p_{1}, q_{1}\right) \neq \emptyset$, $R_{S W} \cap S^{\prime}\left(p_{1}, q_{1}\right) \neq \emptyset$. Thus,

$$
S_{x} \geq \min \left\{\left(p_{1}\right)_{x},\left(q_{1}\right)_{x}\right\} \quad \text { and } \quad W_{y} \geq \min \left\{\left(p_{1}\right)_{y},\left(q_{1}\right)_{y}\right\} .
$$

If $\operatorname{slope}\left(p_{1}, q_{1}\right)>0$, then either $\left(q_{1}\right)_{x}>\left(p_{1}\right)_{x} \geq S_{x}$ or $\left(p_{1}\right)_{y}>\left(q_{1}\right)_{y} \geq W_{y}$. There are no two points of $P$ in the same horizontal or vertical line; thus, $p_{1}=S$ or $q_{1}=W$ by (12). In either case, $S^{\prime}\left(p_{1}, q_{1}\right)$ does not intersect the interior of the region $R_{S W}$, contradicting $S(k, k+1) \cap S^{\prime}\left(p_{1}, q_{1}\right) \neq \emptyset$. Therefore, slope $\left(p_{1}, q_{1}\right)<0$.

Consider the set $P^{\prime}=\left\{i: 1 \leq i \leq p_{1}\right.$ or $\left.q_{1} \leq i \leq n\right\} \subseteq P$. Note that the south, east-, north-, and west-most points of $P^{\prime}$ are $S, p_{1}, q_{1}$, and $W$ respectively, and by (12) we have $(S)_{x}>\left(q_{1}\right)_{x}$ and $(W)_{y}>\left(p_{1}\right)_{y}$. This implies that the northeast region of $P^{\prime}$ only contains the points $p_{1}$ and $q_{1}$. Moreover, only the northeast and the southwest regions of $P^{\prime}$ intersect. This means that $M_{0}=\{S(l, l+1)$ : $l \in P^{\prime}$ and $l$ even $\}$ is a perfect strong matching of $P^{\prime}$ and, therefore, $S(i, i+1) \cap$ $S(j, j+1)=\emptyset$. 

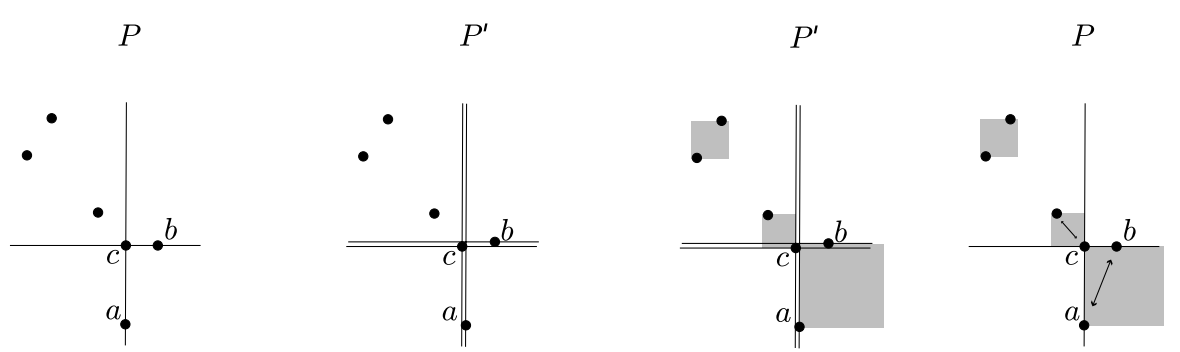

Fig. 12 A point set $P$ with repeated coordinates (left), the perturbed set $P^{\prime}$, and the matchings for $P^{\prime}$ and $P$

\subsection{A Remark on Degeneracies}

We have assumed, for the preceding results, that the points $P$ do not have a repeated $x$ - or $y$-coordinate. Without this assumption, it may be that a perfect matching, even a weak one, does not exist, as shown in the example of Fig. 12 (left), where both $a$ and $b$ can only be matched with $c$.

A natural approach would be to declare that two points can be matched with an object that covers them when no third point is in the interior of the object. However, for a set of points on a horizontal line, the matching graph for squares would then be the complete graph, violating the proximity relationship that the Delaunay graph is expected to have for the $L_{\infty}$ metric.

Another natural and more restrictive extension is as follows. Consider all vertical and horizontal lines defined by the points of $P$, and let $\delta$ be the smallest distance between any two of these lines that are distinct and parallel. Let $\varepsilon$ be an infinitesimal amount with respect to $\delta$, e.g., $\varepsilon=\delta \times n^{-10}$. From $P$, we define a perturbed associated set $P^{\prime}$ as follows. Points $\left(x_{1}, y\right),\left(x_{2}, y\right), \ldots,\left(x_{k}, y\right)$ on a horizontal line, with $x_{1}<x_{2}<\cdots<x_{k}$, are transformed into points $\left(x_{1}, y\right),\left(x_{2}, y+\varepsilon\right), \ldots,\left(x_{k}, y+\right.$ $(k-1) \varepsilon)$, and points $\left(x, y_{1}\right),\left(x, y_{2}\right), \ldots,\left(x, y_{k}\right)$ on a vertical line, with $y_{1}>y_{2}$ $>\cdots>y_{k}$, are transformed into $\left(x, y_{1}\right),\left(x, y_{2}+\varepsilon\right), \ldots,\left(\left(x, y_{k}\right)+(k-1) \varepsilon\right)$. (This is essentially the same perturbation produced by an infinitesimal clockwise rotation of the coordinate axis.) Now the extended matching definition for $P$ is simply what results from applying the original definition to $P^{\prime}$, where no $x$ - or $y$-coordinates are repeated, and, thus, all of our preceding results apply.

With this definition, the matching graph for a set of points on a horizontal line is a path, as is natural. Notice that points that would be matched in $P$ with the original definition are still matched with the extended definition via $P^{\prime}$, and that strong square-matchings in $P^{\prime}$ give squares for $P$ that have disjoint interiors, which is an acceptable definition for strong matching in the extended scenario. An example of matching for the extended definition is shown in Fig. 12. This can be easily reformulated for the $L_{1}$ metric, where repeated points of lines with slope \pm 1 must be avoided. 
Fig. 13 The elements of a set $S$ are $n-1$ points evenly distributed on $C$ and the center of $C$. For $n \geq 74$, this point set does not admit any strong perfect circle-matching

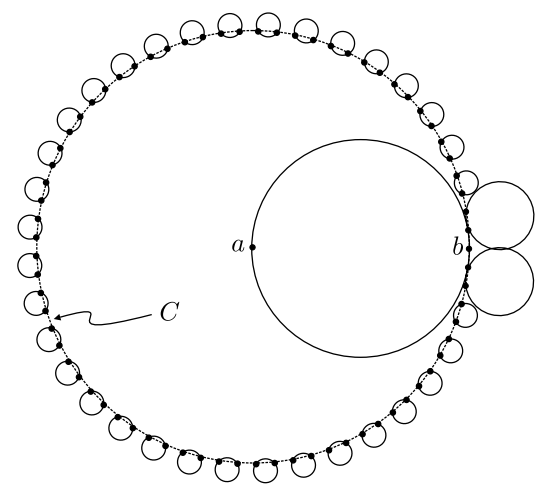

\section{Conclusion}

\subsection{Square-Matchings versus Circle Matchings}

Let us briefly compare the results on matching points using squares with the analogous results using circles; the interested reader is referred to [1] for details.

When $\mathcal{C}$ is the set of all circles in the plane, the graph $G_{\mathcal{C}}(P)$ is the Euclidean $\left(L_{2}\right)$ Delaunay triangulation $D T(P)$; hence, a point set admits a perfect circle-matching if and only if the graph $D T(P)$ contains a perfect matching, which is always the case, as proved by Dillencourt in 1990 [5]. Therefore, while we have had to prove the existence of square-matchings from scratch, the fact that any point set of even cardinality admits a perfect circle-matching is a direct consequence of Dillencourt's result. On the other hand, he also proved that for the $L_{2}$ metric, $D T(P)$ does not contain in general a Hamiltonian path [4], contrary to the situation for the $L_{\infty}$ and the $L_{1}$ metrics, as we have established here.

There are point sets that do not admit strong-circle matchings, as is also the case for strong square-matchings. However, the example described in this paper requires only 10 points, while the smallest example we found for circles requires 74 points (Fig. 13). Similarly, we have shown that, given a point set $P$ with $n \geq 2$ elements, it is always possible to find a strong square-matching of $P$ with $2\left\lceil\frac{n}{5}\right\rceil$ matched points, while for circles, the best fraction we know is that there is a strong circle-matching using at least $2\lceil(n-1) / 8\rceil$ points of $P$.

A final difference that is worth mentioning happens when $P$ is a point set in convex position with an even number of elements. While we have proved that in this situation $P$ always admits a perfect strong square-matching (Theorem 4 ), an example disallowing strong circle-matching is shown in [1].

\subsection{Open Problems}

Since (weak) perfect matchings with circles and isothetic squares are always possible, it is natural to ask which other classes of convex objects have the same property and try to characterize them. On the other hand, we have also shown that perfect strong matchings are not always possible using either circles or squares; hence, it would be interesting to find some nontrivial class of objects that allows them. 
On the computational side, there are also decision and construction problems that are very interesting; in particular, in the time since the conference presentation of our results, Bereg et al. [2] were able to prove that deciding whether a point set $P$ admits a perfect strong square matching is NP-hard, while, given $P$ and a specific combinatorial matching, deciding whether the matching is realizable as a strong square-matching can be done in $O\left(n \log ^{2} n\right)$ time. However, similar problems for circles remain open.

Acknowledgements The research in this paper was initiated at the International Workshop on Combinatorial Geometry at the Departament de Matemàtica Aplicada II, Universitat Politècnica de Catalunya, Jun. 30-Jul. 4, 2003. The authors would like to thank the other workshop participants, namely, Gabriela Araujo, Elsa Omaña-Pulido, Eduardo Rivera-Campo, and Pilar Valencia for helpful discussions.

E. Arkin and J. Mitchell acknowledge support from the National Science Foundation (CCR-0098172, CCF-0431030, CCF-0729019). In addition, J. Mitchell acknowledges support from the US-Israel Binational Science Foundation (grant No. 2000160), NASA Ames Research (NAG2-1620), NSF grants ACI-0328930 and CCF-0528209, and Metron Aviation. Ferran Hurtado is partially supported by grants MEC-MCYT-FEDER BFM2003-00368, MEC MTM2006-01267, DURSI 2001SGR00224, and DURSI 2005SGR00692. Jorge Urrutia is partially supported by MEC MTM2006-03909 and by CONACYT of Mexico grant 37540-A.

\section{References}

1. Ábrego, B.M., Arkin, E., Fernández-Merchant, S., Hurtado, F., Kano, M., Mitchell, J.S.B., Urrutia, J.: Matching points with circles and squares. In: Proc. Japan Conference on Discrete and Computational Geometry 2004. Lecture Notes in Computer Science, vol. 3742, pp. 1-15. Springer, Berlin (2005)

2. Bereg, S., Mutsanas, N., Wolff, A.: Matching points with rectangles and squares. In: Proc. 32nd Int. Conf. on Current Trends in Theory and Practice of Computer Science (SOFSEM'06). Lecture Notes in Computer Science, vol. 3831, pp. 177-186. Springer, Berlin (2006)

3. Czyzowicz, J., Rivera-Campo, E., Urrutia, J., Zaks, J.: Guarding rectangular art galleries. Discrete Math. 50, 149-157 (1994)

4. Dillencourt, M.B.: A non-Hamiltonian, nondegenerate Delaunay triangulation. Inf. Process. Lett. 25(3), 149-151 (1987)

5. Dillencourt, M.B.: Toughness and Delaunay triangulations. Discrete Comput. Geom. 5(6), 575-601 (1990). Preliminary version in Proc. of the 3rd Ann. Symposium on Computational Geometry, Waterloo, pp. 186-194 (1987)

6. Dillencourt, M.B.: Traveling salesman cycles are not always subgraphs of Delaunay triangulations or of minimum weight triangulations. Inf. Process. Lett. 24(5), 339-342 (1987)

7. Dillencourt, M.B.: Finding Hamiltonian cycles in Delaunay triangulations is NP-Complete. Discrete Appl. Math. 64(3), 207-217 (1996)

8. Liotta, G.: Proximity drawings. In: R. Tamassia (ed.) Handbook of Graph Drawing and Visualization. Chapman \& Hall/CRC Press (2009, in press). ISBN 1584884126

9. Liotta, G., Lubiw, A., Meijer, H., Whitesides, S.H.: The rectangle of influence drawability problem. Discrete Comput. Geom. 5(6), 575-601 (1990)

10. Pach, J. (ed.): Towards a Theory of Geometric Graphs. Contemp. Math. Series, vol. 342. Am. Math. Soc., Providence (2004)

11. Tutte, W.T.: A theorem on planar graphs. Trans. Am. Math. Soc. 82, 99-116 (1956) 